\title{
Optimizing VAP scars after childhood cancer treatment: a pilot study
}

\author{
C. M. A. de Bruijn ${ }^{1}$ - F. W. Hoff ${ }^{1}$ - M. M. Bruggeman-Westermann ${ }^{2}$ - J. B. Terra ${ }^{3}$. \\ T. H. van Dijk ${ }^{4}$ - E. S. J. M. de Bont ${ }^{1}$ - A. M. L. Peek ${ }^{1}$
}

Received: 26 January 2017 / Accepted: 7 June 2017 / Published online: 28 June 2017

(C) The Author(s) 2017. This article is an open access publication

\begin{abstract}
Purpose Majority of pediatric cancer patients are treated with chemotherapy using Venous Access Ports (VAP). However, after surgical removal of the VAP prominent scars often remain and standard care is lacking.

Methods Patients $(N=20)$ who were willing to participate were included prior to surgical removal of their VAP. All patients were off therapy at time of VAP removal. Patients had the option to either choose from Dermatix $\AA$, meridian color therapy (MCT), or no additional treatment (NAT). Assessment of scars was done prior to and 3, 6, and 12 months after surgical VAP removal using Patient and Observer Scar Assessment Scales (POSAS) questionnaires. To identify whether Dermatix ${ }^{\circledR}$ or MCT is associated with better scar healing than without additional treatment, Mann-Whitney $U$ tests were used.

Results After 12 months of follow-up, both patients and dermatologists noted VAP scars had healed better after MCT compared to those without treatment $(P=0.010$ for both POSAS patient and POSAS observer). No significant
\end{abstract}

C. M. A. de Bruijn and F. W. Hoff contributed equally to this work

A. M. L. Peek

a.m.1.peek@umcg.nl

1 Department of Pediatric Oncology/Hematology, Beatrix Children's Hospital, University Medical Center Groningen, University of Groningen, PO Box 30.001, 9700 RB Groningen,

Groningen, The Netherlands

2 Practice for Color Meridian Therapy, Groningen, The Netherlands

3 Department of Dermatology, University Medical Center Groningen, University of Groningen, Groningen, The Netherlands

4 Department of Pediatric Surgery, Beatrix Children's Hospital, University Medical Center Groningen, University of Groningen, Groningen, The Netherlands differences were observed between VAP scars after Dermatix ${ }^{\circledR}$ use and those with no treatment.

Conclusions Scar healing after MCT significantly improved, whereas Dermatix ${ }^{\circledR}$ treatment showed no significant differences compared to NAT. To translate this to daily care, a larger prospective study is needed to validate these findings.

Keywords VAP $\cdot$ Scar healing $\cdot$ Pediatric oncology

\section{Introduction}

Majority of pediatric cancer patients are treated with chemotherapy. For optimal administration of long-term intravenous treatment, implementation of venous access ports (VAP) is part of the standard care. In the University Medical Center Groningen, VAP catheters are usually positioned in the subclavian or external jugular vein, whereas the VAP reservoir is positioned below the clavicle. Unfortunately, after removal of the VAP prominent scars often remain, which can be debilitating for patients. A recent study showed that $33 \%$ of the adult childhood cancer survivors reported treatment-related scarring, associated with body imagine disturbance, emotional distress, and reduced quality of life. Moreover, the proportion of patients that reported head/neck scarring was highest among survivors between the age of 10 and 14 years old, which is a critical time for developing self-esteem. [1] Another study reported that $15 \%$ of the psychological problems in survivors of childhood cancer resulted from scar formation. [2]

Information regarding standardized care of VAP scars is not available. In daily practice, treatment options are used to optimize healing of the VAP scar, including meridian color therapy (MCT), and silicone gel (Dermatix $\left.{ }^{\circledR}\right)$. MCT is a treatment based on the reflective zones of the connective tissue, the 
anthroposophical color system, and acupuncture. It restores blockages in energy flows (meridians) by adding color frequencies with silk patches dyed with natural pigments of plants. [3] Despite lack of scientific evidence, patients reported positive results that suggest potential benefits for VAP scar treatment. Likewise, dermatologists experienced positive results with Dermatix ${ }^{\circledR}$ in scar treatment. Dermatix ${ }^{\circledR}$ can be applied easily, and the mechanism is based on recovering skin moisture, restoring skin structures and stimulating collagen production. [4]

Due to lack of evidence-based standardized care for VAP scars, the aim was to assess scar healing in patients who were willing to share their healing process. Patients were enabled to decide themselves between treatment with silicone gel (Dermatix $\left.{ }^{\circledR}\right)$, meridian color therapy (MCT), or no additional treatment (NAT).

\section{Methods}

Twenty pediatric cancer patients with a history of chemotherapy treatment who were willing to share their healing process were followed prior to and after surgical VAP removal between March 2014 and March 2016. Patients had a median age of 7.65 years (range $1.51-15.13)$, and $65 \%(N=13 / 20)$ of the patients were female. All patients were off therapy at time of VAP removal and no local complications (e.g., VAP wound infection or hypertrophic scar formation) were observed. Patients had the option to choose Dermatix ${ }^{\circledR}$, MCT, or NAT after VAP removal. Patients who opted for Dermatix ${ }^{\circledR}$ were advised to start with daily application after the wound had healed superficially. When opting for MCT, patients were counseled by a MCT therapist beforehand and had to apply colored silk bandages at determined meridian points of the skin. Both MCT and Dermatix ${ }^{\circledR}$ were applied at home for at least 6 months. No adjustments were made in addition to the standardized protocol for surgical VAP removal.

Scar healing was assessed prior to and 3, 6, and 12 months after VAP removal using the Patient and Observer Scar Assessment Scales (POSAS). [5] The POSAS scale is divided into two parts: a patient scale and an observer scale. Scales numerically score six items on a measure from one to ten, where a lower score represented better scar recovery. Parents filled out the POSAS questionnaire if patients were $<8$ years. Additionally, photographs of the VAP scars were taken, using a Canon camera (Eos 600D DS126311, Macro lens EF-S $60 \mathrm{~mm}$ ). Two independent dermatologists assessed the scars retrospectively without any knowledge of the scar treatment. Dermatologists were trained to consistently use the POSAS scale. If large intervariability was observed between dermatologists, re-assessment of that particular scar was done.

To identify whether Dermatix ${ }^{\circledR}$ or MCT was associated with better scar healing and to assess whether scars improved within treatment groups after 12 months compared to 3 months, Mann-Whitney $U$ tests were used. Statistical analysis was done using the SPSS statistical software (IBM SPSS Statistics 22) and the figure was generated in R (Version 0.99.484). Intervariability between the two dermatologists was determined using the reliability analysis (Cronbach's alpha).

\section{Results}

Twenty-one scars were analyzed of which one patient had two scars. Eight scars were treated with Dermatix $₫$, seven with MCT, and six with NAT. No statistical differences were observed for gender, duration of the VAP in situ, or age between the treatment groups. Scar assessment showed no intervariability between dermatologists (Cronbach's alpha $=0.933$ ).

Prior to removal of the VAP, the POSAS observer scale showed higher scores for patients who intended to start MCT compared to patients who chose NAT $(P=0.007)$. Strikingly, patients who chose MCT treatment assessed their former scar superior compared to the control group, although this was not significantly $(P=0.086)$. POSAS observer scores showed no differences between patients who chose Dermatix ${ }^{\circledR}$ compared to patients with NAT $(P=0.826)$. Likewise, assessment done by patients using Dermatix ${ }^{\circledR}$ showed no differences compared to patients without treatment $(P=0.061)$. POSAS scores of patients and dermatologists are shown in Fig. 1.

After VAP removal, scar evaluations of patients and observers showed lower POSAS scores for MCT compared to NAT at 6 and 12 months $(P=0.017 ; P=0.042$ at 6 months and $P=0.010 ; P=0.010$ at 12 months for, respectively, patients and observers). Similarly, scars treated with Dermatix ${ }^{\circledR}$ showed lower POSAS patient scores compared to NAT at 3 months $(P=0.022)$, but this was not confirmed by dermatologist' assessment $(P=0.116)$ and did not last after 12 months. Despite superior scar healing for patients using MCT compared to patients with NAT, no significant improvement was seen between $\mathrm{T} 3$ and $\mathrm{T} 12$ within patients using MCT. Also, no improvements over time were seen after Dermatix ${ }^{\circledR}$ application. Fig. 2 shows three examples of scar healing, each representing a different treatment option.

\section{Discussion}

Chemotherapy is still the mainstay of treatment for pediatric cancer patients, and to optimize long-term intravenous administration VAPs are frequently implemented. Although great progress has been achieved in cancer-treatment outcome, little focus has been directed to scar-related consequences of VAP treatment that may negatively affect psychological functioning. This manuscript aimed to compare two potential 
Fig. 1 Scar assessment prior to and at 3,6, and 12 months after surgical VAP removal using the POSAS patient scale (upper) and POSAS observer scale (lower). Patients without additional treatment are shown in light gray, patients using Dermatix® in white and patients using MCT are shown in dark gray. Horizontal bars indicate median scores and a lower score represents better scar recovery. Asterisks (*) indicate significant differences $(P<0.05)$ compared to natural scar recovery

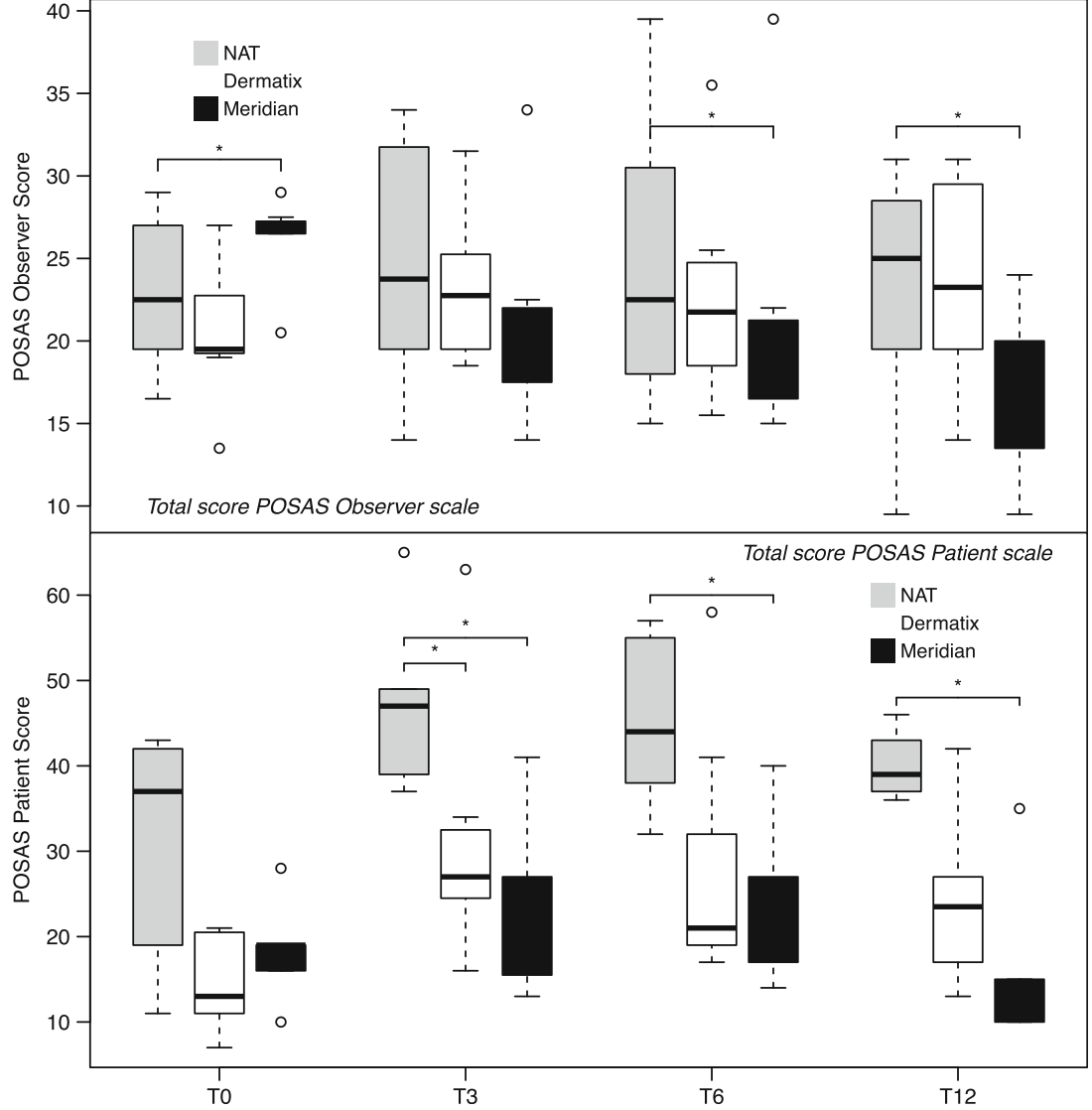

treatments to optimize VAP scar healing in pediatric patients compared to NAT.

The prime discovery in this study was improved scar healing after removal of the VAP in patients that used MCT compared to patients with NAT predicated upon lower POSAS scores. No significant improvement in scar healing was observed after Dermatix ${ }^{\circledR}$ application. The feature that makes this observation strong was scar assessment performed by both patients and dermatologists.

Since patients who chose MCT, assessed their scars more positively before and after VAP removal; this could imply that patients who chose MCT had a more optimistic perception. However, dermatologist did not confirm this finding. They did agree with the improvement of the scars after 6 and 12 months compared to the scars with NAT. Although scar healing after MCT was significantly better compared to NAT, no improvement was seen after 12 months compared to 3 months. This could suggest that the follow-up time was too short to attain optimal scar healing in our patient group.

To translate these outcomes to daily care, a larger study is needed to confirm these findings. More patients should be included, and follow-up time should be increased to ensure
Fig. 2 Scar evaluations at 3 and 12 months after VAP removal. Scar recovery at 3 and 12 months after removal of the VAP is shown for three patients, each representing a different treatment option; natural scar healing, Dermatix ${ }^{\circledR}$, and MCT. All patients agreed upon using their scar photographs

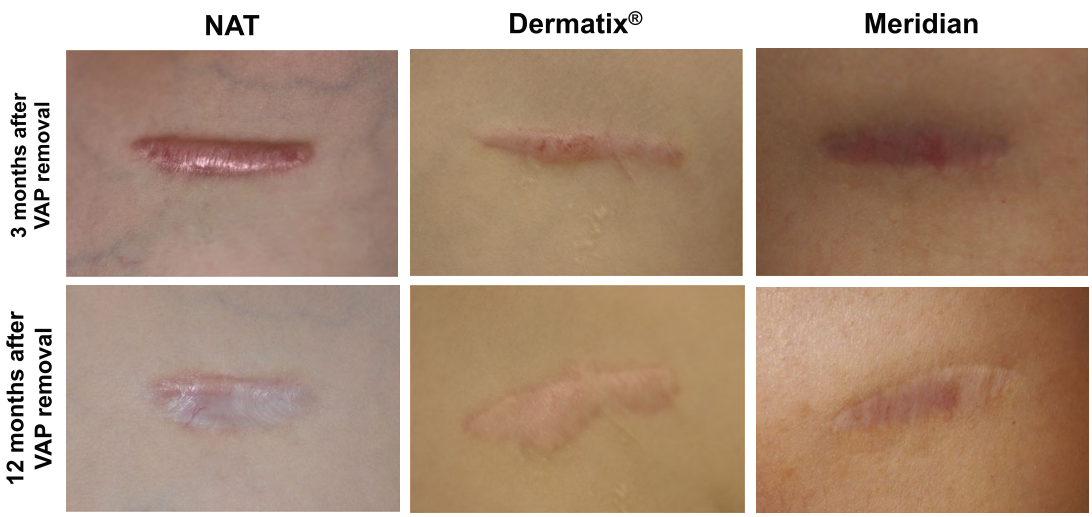


maximal scar-recovery. During time of follow-up, patients were asked to use therapy for at least 6 months. However, no certainty was given according to compliance to therapy, which may have affected our results. Therefore, future studies should thoroughly try to monitor compliance to therapy. Also, a standardized surgery procedure concerning suture material and techniques needs to be incorporated as this may affect scar healing. Moreover, it would be interesting to determine whether inferior scar healing affects the quality of life and whether better scar recovery improves the quality of life. In conclusion, MCT showed more promising results and could be considered for optimization of the scar healing after surgical VAP removal in pediatric cancer patients.

\section{Compliance with ethical standards}

Conflict of interest The authors declare that they have no conflict of interest.

Open Access This article is distributed under the terms of the Creative Commons Attribution-NonCommercial 4.0 International License (http:// creativecommons.org/licenses/by-nc/4.0/), which permits any noncommercial use, distribution, and reproduction in any medium, provided you give appropriate credit to the original author(s) and the source, provide a link to the Creative Commons license, and indicate if changes were made.

\section{References}

1. Kinahan KE, Sharp LK, Seidel K, Leisenring W, Didwania A, Lacouture ME et al (2012 Jul 10) Scarring, disfigurement, and quality of life in long-term survivors of childhood cancer: a report from the childhood cancer survivor study. J Clin Oncol 30(20):2466-2474

2. Pinter AB, Hock A, Kajtar P, Dober I (2003 Jun) Long-term followup of cancer in neonates and infants: a national survey of 142 patients. Pediatr Surg Int 19(4):233-239

3. Van Wijk EPA, Bosman S, Tjeerdema C, Frerich B, van Wijk R. Meridiaan-kleurentherapie. Invloed van zijde met natuurlijke kleurstoffen op de fotonenemissie van de mens. TIG. TIG. Jaarboek Integrale Geneeskunde; 2007. p. 96-111.

4. Murison M, James W (2006) Preliminary evaluation of the efficacy of dermatix silicone gel in the reduction of scar elevation and pigmentation. J Plast Reconstr Aesthet Surg 59(4):437-439

5. Draaijers LJ, Tempelman FR, Botman YA, Tuinebreijer WE, Middelkoop E, Kreis RW et al (2004 Jun) The patient and observer scar assessment scale: a reliable and feasible tool for scar evaluation. Plast Reconstr Surg 113(7):1960-1965 discussion 1966-7 